\title{
On L-Close Sperner Systems
}

\author{
Dániel T. Nagy ${ }^{1} \cdot$ Balázs Patkós ${ }^{1,2}$
}

Received: 8 April 2020/Revised: 11 January 2021 / Accepted: 19 January 2021 / Published online: 12 February 2021

(C) The Author(s) 2021

\section{Abstract}

For a set $L$ of positive integers, a set system $\mathcal{F} \subseteq 2^{[n]}$ is said to be $L$-close Sperner, if for any pair $F, G$ of distinct sets in $\mathcal{F}$ the skew distance $\operatorname{sd}(F, G)=\min \{|F\rangle$ $G||, G \backslash F \mid\}$ belongs to $L$. We reprove an extremal result of Boros, Gurvich, and Milanič on the maximum size of $L$-close Sperner set systems for $L=\{1\}$, generalize it to $|L|=1$, and obtain slightly weaker bounds for arbitrary $L$. We also consider the problem when $L$ might include 0 and reprove a theorem of Frankl, Füredi, and Pach on the size of largest set systems with all skew distances belonging to $L=\{0,1\}$.

Keyword Extremal set systems $\cdot$ Sperner type theorems $\cdot$ Polynomial method

\section{Introduction}

One of the first results of extremal finite set theory is Sperner's theorem [13] that states that if for any pair $F, F^{\prime}$ of distinct sets in a set systems $\mathcal{F} \subseteq 2^{[n]}$ we have $\min \left\{\left|F \backslash F^{\prime}\right|,\left|F^{\prime} \backslash F\right|\right\} \geq 1$, then $|\mathcal{F}| \leq\left(\begin{array}{c}n \\ \lfloor n / 2\rfloor \mid\end{array}\right)$ holds. Set systems with this property are called antichains or Sperner systems. This theorem has lots of generalizations and applications in different areas of mathematics (see the book [7] and Chapter 3 of [11]). Recently, Boros, Gurvich, and Milanič introduced the following notion: given a positive integer $k$, we say that a set system $\mathcal{F}$ is $k$-close

Balázs Patkós

patkos@renyi.hu

Dániel T. Nagy

nagydani@renyi.hu

1 Alfréd Rényi Institute of Mathematics, P.O.B. 127, Budapest 1364, Hungary

2 Laboratory of Combinatorial and Geometric Structures, Moscow Institute of Physics and Technology, Dolgoprudny, Russia 
Sperner if every pair $F, G \in \mathcal{F}$ of distinct sets satisfies $1 \leq \min \{|F \backslash G|||, G \backslash F \mid\} \leq k$. In particular, $\mathcal{F}$ is 1 -close Sperner if every pair $F, G \in \mathcal{F}$ of distinct sets satisfies $\min \{|F \backslash G|||, G \backslash F \mid\}=1$. (The authors used the unfortunate $k$-Sperner term which, throughout the literature, refers to set systems that are union of $k$ many antichains. That is why we decided to use instead the terminology $k$-close Sperner systems.) Boros, Gurvich, and Milanič's motivation to study these set systems comes from computer science: they wanted to compare them to other classes of Sperner systems (see also [4, 6]). They obtained some structural results from which they deduced the following extremal theorem. For a set $F \subseteq[n]=\{1,2, \ldots, n\}$, its characteristic vector $v_{F}$ is a $0-1$ vector of length $n$ with $\left(v_{F}\right)_{i}=1$ if and only if $i \in F$.

Theorem 1.1 (Boros et al. [5]) If the set system $\{\emptyset\} \neq\left\{F_{1}, F_{2} \ldots, F_{m}\right\} \subseteq 2^{[n]}$ is 1close Sperner, then the characteristic vectors $v_{F_{1}}, v_{F_{2}}, \ldots, v_{F_{m}}$ are linearly independent over $\mathbb{R}$. In particular, $m \leq n$.

In this short note, we reprove the extremal part of Theorem 1.1 via a different linear algebraic approach and generalize the result. For a subset $L$ of $[n]$, we say that a set system $\mathcal{F}$ is L-close Sperner if every pair $F, G \in \mathcal{F}$ satisfies $\min \{|F \backslash G|,|G \backslash F|\} \in L$. Note that being $\{k\}$-close Sperner is equivalent to being $k$-close Sperner, and we will use the latter notation. Our first result is the following.

Theorem 1.2 If the set system $\left\{F_{1}, F_{2} \ldots, F_{m}\right\} \subseteq 2^{[n]}$ is L-close Sperner for some $L \subseteq[n]$, then we have $m \leq \sum_{h=0}^{|L|}\left(\begin{array}{l}n \\ h\end{array}\right)$. Furthermore, if $|L|=1$, then $m \leq n$ holds.

Note that if $|L|$ is fixed and $n$ tends to infinity, then the bound is asymptotically sharp as shown by $L=\{1,2, \ldots, k\}$ (i.e. the $k$-close Sperner property) and the set system $\left(\begin{array}{c}{[n]} \\ k\end{array}\right)=\{F \subseteq[n]:|F|=k\}$. Observe also that the inequality $m \leq n$ is sharp for $L=\{1\}$ as shown by the family of singletons, but there exist many other 1-close Sperner systems with $n$ sets: the family of all co-singletons (complements of singletons) is 1-close Sperner. Also, if $A_{1} \cup A_{2}$ is a partition of [ $n$ ], then the family $\left\{\{a\}: a \in A_{1}\right\} \cup\left\{A_{2} \backslash\{a\}: a \in A_{2}\right\}$ is 1-close Sperner and of size $n$. Furthermore, if $L=\{q\}$ for some prime power $q$ and $n=q^{2}+q+1$, then the lines of a projective plane of order $q$ form an $L$-close family of size $n$, so the bound $m \leq n$ is sharp in this case, too.

Apart from Sperner-type theorems, the other much studied area in extremal finite set theory are intersection properties (see e.g. Chapter 2 of [11]). For a set $L$ of integers, a set system $\mathcal{F}$ is said to be $L$-intersecting if for any pair $F, F^{\prime}$ of distinct sets in $\mathcal{F}$ we have $\left|F \cap F^{\prime}\right| \in L$. Frankl and Wilson [10] proved the same upper bound $\sum_{h=0}^{|L|}\left(\begin{array}{l}n \\ h\end{array}\right)$ on the size of $L$-intersecting set systems. Frankl and Wilson used higher incidence matrices to prove their result, but later the polynomial method (see $[1,2])$ turned out to be very effective in obtaining $L$-intersection theorems. In the proof of the furthermore part of Theorem 1.2, an additional idea due to Blokhuis [3] will be used. 
We will need the following well-known lemma, we include the proof for sake of completeness. For any field $\mathbb{F}$, we denote by $\mathbb{F}^{n}[x]$ the vector space over $\mathbb{F}$ of polynomials of $n$ variables with coefficients from $\mathbb{F}$.

Lemma 1.3 Let $p_{1}(x), p_{2}(x), \ldots, p_{m}(x) \in \mathbb{F}^{n}[x]$ be polynomials and $v_{1}, v_{2}, \ldots, v_{m} \in$ $\mathbb{F}^{n}$ be vectors such that $p_{i}\left(v_{i}\right) \neq 0$ and $p_{i}\left(v_{j}\right)=0$ holds for all $1 \leq j<i \leq m$. Then the polynomials are linearly independent.

Proof Suppose that $\sum_{i=1}^{m} c_{i} p_{i}(x)=0$. As $p_{i}\left(v_{1}\right)=0$ for all $1<i$ we obtain $c_{1} p_{1}\left(v_{1}\right)=0$ and therefore $c_{1}=0$ holds. We proceed by induction on $j$. If $c_{h}=0$ holds for all $h<j$, then using this and $p_{i}\left(v_{j}\right)=0$ for all $i>j$, we obtain $c_{j} p_{j}\left(v_{j}\right)=0$ and therefore $c_{j}=0$.

Results on $L$-intersecting families had some geometric consequences on point sets in $\mathbb{R}^{n}$ defining only a few distances, in particular on set systems $\mathcal{F}$ with only a few Hamming distances. The skew distance $\operatorname{sd}(F, G):=\min \{|F \backslash G|,|G \backslash F|\}$ does not define a metric space on $2^{[n]}$ as $s d(F, G)=0$ holds if and only if $F \subseteq G$ or $G \subseteq F$ and one can easily find triples for which the triangle inequality fails: if $A$ is the set of even integers in $[n], C$ is the set of odd integers in $[n]$, and $B=\{1,2\}$, then $\lfloor n / 2\rfloor=s d(A, C) / \leq s d(A, B)+s d(B, C)=1+1$

One can also investigate the case when $L$ includes 0 . Then set systems with the required property are not necessarily Sperner, so we will say that $\mathcal{F}$ is $L$-skew distance (or $L$-sd for short) if $s d(A, B) \in L$ for all pairs of distinct sets $A, B \in \mathcal{F}$. We will write $\operatorname{ex}_{s d}(n, L)$ to denote the largest size of an $L$-skew distance system $\mathcal{F} \subseteq 2^{[n]}$. Observe that $e x_{s d}(n,\{0\})$ asks for the maximum size of a chain in $2^{[n]}$ which is obviously $n+1$. This shows that the furthermore part of Theorem 1.2 does not remain valid in this case. In a different context Frankl, Füredi, and Pach considered the case $L=\{0,1, \ldots, t\}$. They considered the following construction: let $\emptyset=C_{0} \subsetneq C_{1} \subsetneq C_{2} \subsetneq \ldots \subsetneq C_{n}=[n]$ be a maximal chain and let

$$
\mathcal{F}_{n, t}=\left\{F: C_{|F|-t} \subset F\right\} \cup\{F:|F| \leq \text { tor }|F| \geq n-t\} .
$$

The size of $\mathcal{F}_{n, t}$ is $\left(\begin{array}{c}n \\ t+1\end{array}\right)-\left(\begin{array}{c}2 t+1 \\ t+1\end{array}\right)+2 \sum_{i=0}^{t}\left(\begin{array}{c}n \\ i\end{array}\right)$ and clearly $\mathcal{F}_{n, t}$ is $\{0,1, \ldots, t\}$-sd. This gives the lower bounds in the following results.

Theorem 1.4 (Frankl et al. [9]) If $n \geq 3$, we have ex $(n,\{0,1\})=\left(\begin{array}{l}n \\ 2\end{array}\right)+2 n-1$.

Theorem 1.5 (Frankl et al. [9]) For any $n, t$ with $n \geq 2(t+2)$, we have

$$
\begin{aligned}
& \left(\begin{array}{c}
n \\
t+1
\end{array}\right)-\left(\begin{array}{c}
2 t+1 \\
t+1
\end{array}\right)+2 \sum_{i=0}^{t}\left(\begin{array}{c}
n \\
i
\end{array}\right) \leq e x_{s d}(n,\{0,1, \ldots, t\})<\left(\begin{array}{c}
n \\
t+1
\end{array}\right)+ \\
& 5(t+1)^{2}\left(\begin{array}{c}
n \\
t
\end{array}\right) .
\end{aligned}
$$

The authors of [9] conjectured that the lower bound is tight in Theorem 1.5 for large enough $n$. (There are larger constructions for small $n$.) We will give a simple, new proof of Theorem 1.4 that proceeds by induction. 


\section{Proof and Remarks}

We start by introducing some notation. For two vectors, $u, v$ of length $n$ we denote their scalar product $\sum_{i=1}^{n} u_{i} v_{i}$ by $u \cdot v$. We will often use the fact that for any pair $F, G$ of sets we have $v_{F} \cdot v_{G}=|F \cap G|$. We will also use that $\min \{|F \backslash G|, \mid G \backslash$ $F \mid\}=|F \backslash G|$ if and only if $|F| \leq|G|$ holds.

For two sets $F, L \subseteq[n]$ we define the polynomial $p_{F, L}^{\prime} \in \mathbb{R}^{n}[x]$ as

$$
p_{F, L}^{\prime}(x)=\prod_{h \in L}\left(|F|-v_{F} \cdot x-h\right) .
$$

We obtain $p_{F, L}(x)$ from $p_{F, L}^{\prime}(x)$ by replacing every $x_{i}^{t}$ term by $x_{i}$ for every $t \geq 2$ and $i=1,2, \ldots, n$. As $0=0^{t}$ and $1=1^{t}$ for any $t \geq 2$, we have $p_{F, L}\left(v_{G}\right)=p_{F, L}^{\prime}\left(v_{G}\right)=\prod_{h \in L}(|F \backslash G|-h)$. Finally, observe that the polynomials $p_{F, L}(x)$ all belong to the subspace $M_{|L|}$ of $\mathbb{R}^{n}[x]$ spanned by $\left\{x_{i_{1}} x_{i_{2}} \ldots x_{i_{l}}: 0 \leq l \leq|L|, i_{1}<i_{2}<\ldots<i_{l}\right\}$, where $l=0$ refers to the constant 1 polynomial 1. Note that $\operatorname{dim}\left(M_{|L|}\right)=\sum_{i=0}^{|L|}\left(\begin{array}{c}n \\ i\end{array}\right)$.

Based on the above, Theorem 1.2 is an immediate consequence of the next result.

Theorem 2.1 If the set system $\left\{F_{1}, F_{2} \ldots, F_{m}\right\} \subseteq 2^{[n]}$ is L-close Sperner, then the polynomials $p_{F_{1}, L}(x), p_{F_{2}, L}(x), \ldots, p_{F_{m}, L}(x)$ are linearly independent in $\mathbb{R}^{n}[x]$. In particular, $m \leq \sum_{h=0}^{|L|}\left(\begin{array}{l}n \\ h\end{array}\right)$. Moreover, if $|L|=1$ and $\left\{F_{1}, F_{2} \ldots, F_{m}\right\} \neq\{\emptyset\}$, then the polynomials $p_{F_{1}, L}(x), p_{F_{2}, L}(x), \ldots, p_{F_{m}, L}(x)$ are linearly independent in $\mathbb{R}^{n}[x]$ even together with 1. In particular, $m \leq n$.

Proof We claim that if $F_{1}, F_{2}, \ldots, F_{m}$ are listed in a non-increasing order according to the sizes of the sets, then the polynomials $p_{F_{1}, L}(x), p_{F_{2}, L}(x), \ldots, p_{F_{m}, L}(x)$ and the characteristic vectors $v_{F_{1}}, v_{F_{2}}, \ldots, v_{F_{m}}$ satisfy the conditions of Lemma 1.3. Indeed, for any $G \subseteq[n]$ we have $p_{F, k}(G)=\prod_{h \in L}(|F|-|F \cap G|-h)=\prod_{h \in L}(|F \backslash G|-h)$. Therefore $p_{F, L}\left(v_{F}\right) \neq 0$ holds for any $F \subseteq[n]$, while if $\left|F_{j}\right| \leq\left|F_{i}\right|$, then the $L$-close Sperner property ensures $\left|F_{i} \backslash F_{j}\right| \in L$ and thus $p_{F_{j}, L}\left(v_{F_{i}}\right)=0$.

To prove the moreover part, let $L=\{s\}, \mathcal{F}=\left\{F_{1}, F_{2}, \ldots, F_{m}\right\}$ and let us suppose towards a contradiction that $\mathbf{1}=\sum_{i=1}^{m} c_{F_{i}} p_{F_{i}, L}(x)$ holds for some reals $c_{F_{i}}$. We claim that if $\left|F_{i}\right|=\left|F_{j}\right|$, then $c_{F_{i}}=c_{F_{j}}$ holds and all coefficients are negative. Observe that for any $F \in \mathcal{F}$ using the $L$-close Sperner property we have

$$
1=c_{F} p_{F, L}\left(v_{F}\right)+\sum_{F^{\prime} \in \mathcal{F}\left|F^{\prime}\right|>|F|} c_{F^{\prime}} p_{F^{\prime}, L}\left(v_{F}\right),
$$

and $p_{F, L}\left(v_{F}\right)=-s$ for all $F$. In particular, if $F$ is of maximum size in $\mathcal{F}$, then $c_{F}=-\frac{1}{s}$ holds. Let $m_{j}$ denote $|\{F \in \mathcal{F}:|F|=j\}|$ and $c_{j}$ denote the value of $c_{F}$ for all $F \in \mathcal{F}$ of size $j$ - once this is proved. By the above, if $j^{*}$ is the maximum size among sets in $\mathcal{F}$, then $c_{j^{*}}$ exists. Suppose that for some $i$ we have proved the existence of $c_{j}$ for all $j$ with $i<j \leq j^{*}$. If there is no set in $\mathcal{F}$ of size $i$, there is nothing 
to prove. If $|F|=i$, then using (1) and the fact $p_{F^{\prime}, L}\left(v_{F}\right)=\left|F^{\prime}\right|-|F|+s-s=$ $\left|F^{\prime}\right|-|F|$ provided $\left|F^{\prime}\right| \geq|F|$, we obtain

$$
1=c_{F} p_{F, L}\left(v_{F}\right)+\sum_{F^{\prime} \in \mathcal{F}\left|F^{\prime}\right|>|F|} c_{F^{\prime}} p_{F^{\prime}, L}\left(v_{F}\right)=-s c_{F}+\sum_{j>i} c_{j} m_{j}(j-i) .
$$

This shows that $c_{F}$ does not depend on $F$ but only on $|F|$ as claimed. Moreover, as $s$, $m_{j}, j-i$ are all non-negative and, by induction, all $c_{j}$ are negative, then in order to satisfy (2), we must have that $c_{i}$ is negative as well. So we proved that all $c_{j}$ 's are negative. But this contradicts $\mathbf{1}=\sum_{i=1}^{m} c_{F_{i}} p_{F_{i}, L}(x)$, as on the right hand side all coefficients of the variables are positive, so they cannot cancel. (If there are variables. This is where the condition $\left\{F_{1}, F_{2} \ldots, F_{m}\right\} \neq\{\emptyset\}$ is used.)

Using the original "push-to-the-middle" argument of Sperner, it is not hard to prove that for any $k$-close Sperner system $\mathcal{F} \subseteq 2^{[n]}$, there exists another one $\mathcal{F}^{\prime} \subseteq 2^{[n]}$ with $|\mathcal{F}|=\left|\mathcal{F}^{\prime}\right|$ and $\mathcal{F}^{\prime}$ containing sets of size between $k$ and $n-k$. Is it true that for such set systems we have $\left\langle p_{F,[k]}: F \in \mathcal{F}^{\prime}\right\rangle \cap M_{k-1}=\{\mathbf{0}\}$ ? This would imply $\operatorname{ex}_{s d}(n,[k])=\left(\begin{array}{l}n \\ k\end{array}\right)$.

Let us now turn to the proof of Theorem 1.4.

Proof of Theorem 1.4 The lower bound is given by the special case $t=1$ of the construction given above Theorem 1.4. It remains to prove the upper bound.

We will prove that a $\{0,1\}$-sd system $\mathcal{F} \subseteq 2^{[n]}$ is of size at most $\left(\begin{array}{l}n \\ 2\end{array}\right)+2 n-1$ by induction on $n$. Since $\left(\begin{array}{l}3 \\ 2\end{array}\right)+2 \cdot 3-1=2^{3}$, the statement is trivially true for $n=3$. Now assume that $n \geq 4$ and we have already proved the statement for $n-1$.

Consider the uniform systems $\mathcal{F}_{i}=\{F \in \mathcal{F}:|F|=i\}$ that are 1-close Sperner. We will define a representative set $C_{i}$ for all nonempty levels. If $\left|\mathcal{F}_{i}\right| \geq 3$, it is an exercise for the reader (see Lemma 19 in [5]) to see that there exists a set $C_{i}$ either

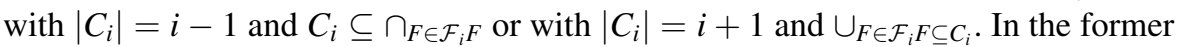
case we say that $\mathcal{F}_{i}$ is of type $\vee$, in the latter case we say that $\mathcal{F}_{i}$ is of type $\wedge$. If $\left|\mathcal{F}_{i}\right|=2$, then we select one of the two sets to be $C_{i}$. If $\left|\mathcal{F}_{i}\right|=1$, then $C_{i}$ is the only set in $\mathcal{F}_{i}$. Finally, if $\mathcal{F}_{i}=\emptyset$, then $C_{i}$ is undefined.

Claim 2.2 If $i<j$ and $\left|\mathcal{F}_{i}\right|,\left|\mathcal{F}_{j}\right|>0$ then $\left|C_{i} \backslash C_{j}\right| \leq 1$.

Proof Assume that there are two different elements $a, b$ such that $a, b \in C_{i}$ but $a, b \notin C_{j}$. It follows from the definition of the representative sets, that there are sets $F_{i} \in \mathcal{F}_{i}$ and $F_{j} \in \mathcal{F}_{j}$ such that $a, b \in F_{i}$ and $a, b \notin F_{j}$. (This is trivial for levels with one or two sets. If there are 3 or more sets then at most two of them can be wrong.)

Let $C_{p_{1}}, C_{p_{2}}, \ldots C_{p_{t}}\left(p_{1}<\ldots<p_{t}\right)$ denote the representative sets of the nonempty levels among $\mathcal{F}_{1}, \mathcal{F}_{2}, \ldots \mathcal{F}_{n-1}$. Since 


$$
\left|\bigcup_{i=1}^{t-1} C_{p_{i}} \backslash C_{p_{i+1}}\right| \leq \sum_{i=1}^{t-1}\left|C_{p_{i}} \backslash C_{p_{i+1}}\right| \leq t-1 \leq n-2,
$$

there will be an element $x \in[n]$ such that $x \notin C_{p_{i}} \backslash C_{p_{i+1}}$ for any $p_{i}$. This implies that there are no nonempty levels $\mathcal{F}_{i}$ and $\mathcal{F}_{j}$ such that $i<j, x \in C_{i}$ but $x \notin C_{j}$. Rearranging the names of the elements, we may assume that $x=n$.

Now we define two families in $2^{[n-1]}$, let

$$
\mathcal{G}=\{F \backslash\{n\} \mid F \in \mathcal{F}\}, \mathcal{H}=\left\{H \in 2^{[n-1]} \mid H, H \cup\{n\} \in \mathcal{F}\right\} .
$$

Note that $|\mathcal{F}|=|\mathcal{G}|+|\mathcal{H}|$. Since $\mathcal{G}$ is a $\{0,1\}$-sd system in $2^{[n-1]}$, we get an upper bound on its size by induction. We will examine $\mathcal{H}$ to bound its size as well.

Claim 2.3 If $A, B \in \mathcal{H}$ and $|A|<|B|$ then $A \subset B$.

Proof By the definition of $\mathcal{H}$, we get that $A \cup\{n\} \in \mathcal{F}$ and $n \notin B$. Since $\mathcal{F}$ is a $\{0,1\}$-sd system, $1 \geq|(A \cup\{n\}) \backslash B|=|A \backslash B|+1$. Therefore we have $|A \backslash B|=0$ or equivalently $A \subset B$.

Claim 2.4 There is at most one level in $\mathcal{H}$ with two or more sets in it.

Proof Assume that there are two sets of size $i$ and two sets of size $j(i<j)$ in $\mathcal{H}$. Then in $\mathcal{F}$ there are two sets of size $i+1$ containing $n$ and two sets of size $j$ that do not contain $n$. From the definition of the representative sets follows that $n \in C_{i+1}$ but $n \notin C_{j}$. This is an outright contradiction if $i+1=j$. If $i+1<j$, it contradicts the special property of the element $n$ established earlier.

Claim $2.5|\mathcal{H}| \leq n+1$.

Proof Let $\mathcal{H}_{i}=\{H \in \mathcal{H}:|H|=i\}$ for all $i=0,1, \ldots, n-1$. If there is no $i$ such that $\left|\mathcal{H}_{i}\right|>1$, then $|\mathcal{H}| \leq n$. Assume that $\left|\mathcal{H}_{t}\right|=k>1$. By Claim 2.4, this is the only level with more than one set. If the level $\mathcal{H}_{t}$ is of type $\vee$, then the union of its sets is of size $t+k-1$. Claim 2.3 implies that all sets $H \in \mathcal{H},|H|>t$ must contain this union, therefore the levels $\mathcal{H}_{t+1}, \mathcal{H}_{t+2}, \ldots, \mathcal{H}_{t+k-2}$ are all empty. If $\mathcal{H}_{t}$ is of type $\wedge$, then the intersection of its sets is of size $t-k+1$. Claim 2.3 implies that all sets $H \in \mathcal{H},|H|<t$ must be subsets of this intersection, therefore the levels $\mathcal{H}_{t-k+2}$, $\mathcal{H}_{t-k+3}, \ldots, \mathcal{H}_{t-1}$ are all empty. In either case we get that $|\mathcal{H}| \leq k+(k-2) \cdot 0+(n-k+1) \cdot 1=n+1$.

Now we can complete the proof of the theorem:

$$
|\mathcal{F}|=|\mathcal{G}|+|\mathcal{H}| \leq\left(\begin{array}{c}
n-1 \\
2
\end{array}\right)+2(n-1)-1+n+1=\left(\begin{array}{l}
n \\
2
\end{array}\right)+2 n-1
$$

Let us make two final remarks.

- Observe that for the set $L_{\ell}=\{\ell+1, \ell+2, \ldots, n\}$ a system $\mathcal{F} \subseteq 2^{[n]}$ is $L_{\ell}$-close Sperner if and only if for every $\ell$-subset $Y$ of $[n]$, the trace 
$\mathcal{F}_{[n] \backslash Y}=\{F \backslash Y: F \in \mathcal{F}\}$ is Sperner. Set systems with this property are called $(n-\ell)$-trace Sperner and results on the maximum size of such systems can be found in Sect. 4 of [12].

- A natural generalization arises in $Q^{n}=\{0,1, \ldots, q-1\}^{n}$. One can partially order $Q^{n}$ by $a \leq b$ if and only if $a_{i} \leq b_{i}$ for all $i=1,2, \ldots, n$. We say that $A \subseteq\{0,1, \ldots, q-1\}^{n}$ is $L$-close Sperner for some subset $L \subseteq[n]$ if for any distinct $a, b \in A$ we have $\operatorname{sd}(a, b):=\min \left\{\left|\left\{i: a_{i}<b_{i}\right\}\right|,\left|\left\{i: a_{i}>b_{i}\right\}\right|\right\} \in L$. One can ask for the largest number of points in an $L$-close Sperner set $A \subseteq Q^{n}$. Here is a construction for 1-close Sperner set: for $2 \leq i \leq n, 1 \leq h \leq q-$ 1 let $\left(v_{i, h}\right)_{i}=h,\left(v_{i, h}\right)_{1}=q-h+1$ and $\left(v_{i, h}\right)_{j}=0$ if $j \neq i, 1$. Then it is easy to verify that $\left\{v_{i, h}: 2 \leq i \leq n, 1 \leq h \leq q-1\right\}$ is 1 -close Sperner of size $(q-1)(n-1)$.

An easy upper bound on the most number of points in $Q^{n}$ that form an 1-close Sperner system is $O_{q}\left(n^{q-1}\right)$. To see this, for any $a=\left\{a_{1}, a_{2}, \ldots a_{n}\right\} \in Q^{n}$ let us define the set $F_{a} \subseteq[(q-1) n]$ as follows.

$$
F_{a}:=\bigcup_{i=1}^{n} \bigcup_{j=1}^{a_{i}}\{(q-1)(i-1)+j\}
$$

If $A \subseteq Q^{n}$ is 1-close Sperner, then $A^{\prime}=\left\{F_{a} \mid a \in A\right\} \subset 2^{[(q-1) n]}$ will be $\{1,2, \ldots q-1\}$-close Sperner. Theorem 1.2 implies

$$
|A|=\left|A^{\prime}\right| \leq \sum_{h=0}^{q-1}\left(\begin{array}{c}
(q-1) n \\
h
\end{array}\right)=O_{q}\left(n^{q-1}\right) .
$$

We conjecture that for any $q$ there exists a constant $C_{q}$ such that the maximum number of points in $Q^{n}$ that form a 1-close Sperner system is at most $C_{q} n$.

Acknowledgements We thank an anonymous referee for the valuable remarks that improved the presentation of the paper.

Open Access This article is licensed under a Creative Commons Attribution 4.0 International License, which permits use, sharing, adaptation, distribution and reproduction in any medium or format, as long as you give appropriate credit to the original author(s) and the source, provide a link to the Creative Commons licence, and indicate if changes were made. The images or other third party material in this article are included in the article's Creative Commons licence, unless indicated otherwise in a credit line to the material. If material is not included in the article's Creative Commons licence and your intended use is not permitted by statutory regulation or exceeds the permitted use, you will need to obtain permission directly from the copyright holder. To view a copy of this licence, visit http:// creativecommons.org/licenses/by/4.0/.

Funding Open Access funding provided by ELKH Alfréd Rényi Institute of Mathematics. The research of Nagy was supported by the National Research, Development and Innovation Office-NKFIH under the grants FK 132060 and K 132696. The research of Patkós was supported partially by the grant of Russian Government N 075-15-2019-1926 and by the National Research, Development and Innovation OfficeNKFIH under the grants FK 132060 and SNN 129364. 


\section{References}

1. Alon, N., Babai, L., Suzuki, M.: Multilinear polynomials and Frankl-Ray-Chaudhuri-Wilson type intersection theorems. J. Comb. Theory Ser. A 58(2), 165-180 (1991)

2. Babai, L., Frankl, P.: Linear algebra methods in combinatorics: with applications to geometry and computer science. Department of Computer Science, University of Chicago (1992)

3. Blokhuis, A.: A new upper bound for the cardinality of 2-distance sets in Euclidean space. Ann. Discrete Math. 20, 65-66 (1984)

4. Boros, E., Gurvich, V., Milanič, M.: Characterizing and decomposing classes of threshold, split, and bipartite graphs via 1-sperner hypergraphs. J. Graph Theory. https://doi.org/10.1002/jgt.22529. arXiv: 1805.03405

5. Boros, E., Gurvich, V., Milanič, M.: Decomposing 1-sperner hypergraphs. Electron. J. Comb. 26(3), 3.18 (2019)

6. Chiarelli, N., Milanič, M.: Linear separation of connected dominating sets in graphs. ARS Math. Contemp. 16, 487-525 (2019)

7. Engel, K.: Sperner theory, vol. 65. Cambridge University Press, Cambridge (1997)

8. Erdös, P., Ko, C., Rado, R.: Intersection theorems for systems of finite sets. Q. J. Math. Oxf. Ser. 12, 313-320 (1961)

9. Frankl, P., Füredi, Z., Pach, J.: Bounding one-way differences. Graphs Comb. 3(1), 341-347 (1987)

10. Frank1, P., Wilson, R.M.: Intersection theorems with geometric consequences. Combinatorica 1, 357-368 (1981)

11. Gerbner, D., Patkós, B.: Extremal finite set theory. CRC Press, Boca Raton (2018)

12. Patkós, B.: $l$-trace $k$-sperner families. J. Comb. Theory Ser. A 116, 1047-1055 (2009)

13. Sperner, E.: Ein Satz über Untermengen einer endlichen Menge. Math. Z. 27, 544-548 (1928)

Publisher's Note Springer Nature remains neutral with regard to jurisdictional claims in published maps and institutional affiliations. 